\title{
THE EFFECT OF IMPLEMENTING OF NONLINEAR FILTERS FOR ENHANCING MEDICAL IMAGES USING MATLAB
}

\author{
Mohamed Y. Adam ${ }^{1}$, Dr Mozamel M. Saeed ${ }^{2}$, Prof. Dr Al Samani A. Ahmed ${ }^{3}$ \\ ${ }^{1}$ king Saud University, TrainingandCommunity Service Center, KSA \\ ${ }^{2}$ prince Sattam Bin Abdul-Aziz University, collage of science, Dept. of computer science, \\ KSA \\ ${ }^{3}$ Al Neelain University collage of computer science \& information technology, Dept. of \\ computer science, Sudan
}

\begin{abstract}
Although this huge development in medical imaging tools, we find that there are some human mistakes in the process of filming medical images, where some errors result in distortions in the image and change some medical image properties which affect thedisease diagnosis correctly.Medical images are one of the fundamental images, because they are used in the most sensitive field which is a medical field. The objective of the study is to identify the effect of implement non-linear filters in enhancingmedical images, using the strongest and most popular program MATLAB, and because of its advantages in image processing. After implementation the researcher concluded that we will get the best result for medical image enhancement by using median filter which is one of the non-linear filters, non-linear filters implemented using Matlab functions.
\end{abstract}

\section{KEYWORDS}

median filter, noise reduction, maximum filter, minimum filter, enhancement, non-linear filters.

\section{INTRODUCTION}

Digital Image Processing means using digital computers to correct the appearance of digital images, correcting the appearance include many activities for example remove noise, smoothing or sharpening the image, improve the contrast of the image, remove blurring which occurred during image acquisition. There are many filter techniques, which use to enhance images, and the enhancement is focus of improving the quality of the image or to obtain certain details. Also filtering can be used to remove unwanted noise. Certainly in medical image we find salt and pepper noise.

There is a big collection of functions in MATLABtoolboxthatuse to implement different types of filters. Some of them are needed in the process of image adjustments and others are responsible for object recognition. Examples of linear and nonlinear filter including their effects are presented in Table1. (Ozimek, lectures from Digital image processing, 2010d) 


\begin{tabular}{|c|c|c|}
\hline Category & Filter & Effect \\
\hline \multirow{5}{*}{ LINEAR } & High-pass filter & $\begin{array}{c}\text { Sharpening } \\
\text { Underlining contours }\end{array}$ \\
\hline & Low-pass filter & $\begin{array}{l}\text { Reduction of the noise, } \\
\text { Smoothing out }\end{array}$ \\
\hline & Laplace's filter & Sharpening \\
\hline & Edge detection filter & \multirow{2}{*}{$\begin{array}{l}\text { Detection of all directions } \\
\text { Edges and corners }\end{array}$} \\
\hline & $\begin{array}{l}\text { Corner detection } \\
\text { filter }\end{array}$ & \\
\hline \multirow{3}{*}{$\begin{array}{l}\text { NON- } \\
\text { LINEAR }\end{array}$} & Median filter & $\begin{array}{c}\text { Noise reduction without } \\
\text { Blur effect }\end{array}$ \\
\hline & Minimum filter & $\begin{array}{l}\text { Decrease the brightness of } \\
\text { the edge objects }\end{array}$ \\
\hline & Maximum filter & $\begin{array}{l}\text { Increase the brightness of } \\
\text { the edge objects }\end{array}$ \\
\hline
\end{tabular}

Table 1. Examples of image enhancing filters

\section{Image Processing Technique}

The main categories of digital image processing techniques include

- Image generation

- Image enhancement

- Image restoration.

Image generation is the most popular digital image processing techniques that use to convert an image into some sort of ordered layout. For example, the reflection created when scanner pick up an image, by digitizing this reflection the result is a series of pixels. By the result the generation techniques help project and recognize a scanned image.

Image enhancement is the process that applied to an image so that the result is more suitable than the original for a specific application.The image properties and the image source will be taking into account while enhancement process, so the techniques that use to enhance x-ray may not be suitable for other kind of image.

Image restoration is an area that also deals with improving the appearance of an image. So enhancement and restoration both are using to improve image appearance, but the image restoration is objective while image enhancement is a subjective process.

\section{IMAGE ENHANCEMENT TeChNiQUe}

The main objective of image enhancement is to improve the quality of the image.Certainly in medical images we will face with more of the following problems:

- The resolution is low.

- Noise high level.

- Contrast is low. 
- geometric deformations;

-presence of imaging artifacts.

The imperfections above can be causedwhile filming the imagefor example X-rays may be low contrast for soft tissues, ultrasound produces very noisy images, and metallic implants willcause imaging artifacts in MRI or the result of a deliberate trade-off during acquisition. But in this study we are focus on the image processing only and not be concerned with the challenging problem of designing optimal procedures for their acquisition

\section{a. NONLINEAR FILTERING}

There are a number of techniques used to remove distortions of images, where the techniques of nonlinear filters is the most important techniques for their properties, so it does not change the properties of the image but remove distortions to enhance image clarity and effectiveness. The linear filters rely on identifying noise and then removed and replaced by the neighborhood points according to the standard, while other points remain unchanged. The most important nonlinear filters aremedian filter, minimum filter, and maximum filter, and each one of these filters characteristics and advantages

\section{b. Median Filtering}

Median filter is one of the most important from nonlinear filters, it is the popular technique which use to remove noise certainly the impulsive-type noise, and by the way it has the ability of preserving image details. The median filter rely on identifying noise and then removed and replaced by the median of neighborhood pixels, while other points remain unchanged. The median pixel means the middle pixel value witch considered after sorting all pixel values from surrounding neighborhood pixels numerically.

In median filter, the pixel value of a point $p$ is replaced by the median of pixel value of 8neighbourhood of a point 'p'. Therefore, we can generate the median filter by the following function:

$$
g(p)=\operatorname{median}\left\{f(p) \text {, where } p \in N_{8}(p)\right\}
$$

- The median value will replace the central pixel according to brightness of the neighbouring pixels.

- When some individual pixels have outliers values, noise will raise out such as shot noise or impulse noise, So median filter can do a good job of removing such noises.

As we can see in figure 1 blow, we can calculate the median value of pixel neighbourhood of 150 , and then is replaced with the median of surrounding pixels value that is 124 . Here we use A3x3 square neighbourhood 


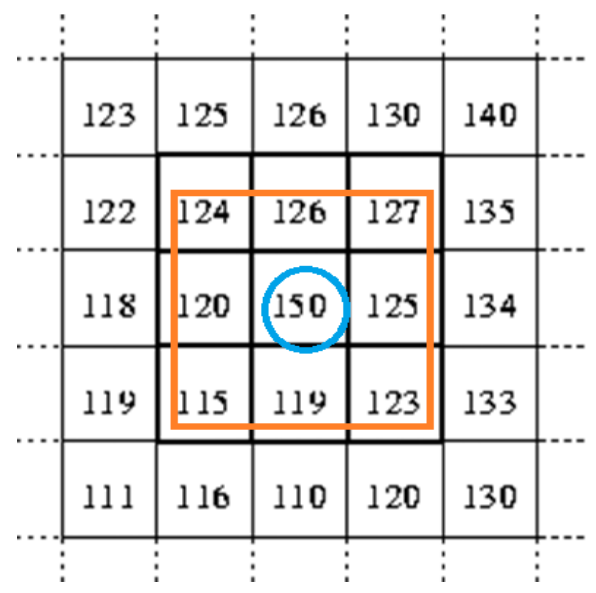

Figure 1. Neighbourhood values

Neighborhood values:

$115,119,120,123,124,125,126,127,150$

Median value: 124

The advantages of median filter:

-Since output values available from the present neighborhood, the contrast will not be change. -Boundaries dose not shift in median filtering, that can happenwith other filters.

-The outliers values will be removed, because the median is less sensitive to the extreme values than the mean.

\section{c. Minimum Filter}

The most effective filter that use to remove positive outline noise is minimum filter, and it'suse also in the computational of binary morphological erosion. Minimum filter defined as:

$$
\operatorname{Minimum}(A)=\min [A(x+i, y+j)],
$$

Where the coordinate $x+I, y+j$ is defined over the image A and the coordinate $I, j$ is defined over the mask $\mathrm{M}$. The mask $\mathrm{M}$ determines which pixels are used in the minimum calculation.

In order to find the darkest points in an image:

First, find the minimum value in the area encompassed by the filter and then reduces the salt noise because of the minimumoperation;finally, the 0th percentile filter is min filter.

\section{d. MaXimum Filter}

The most effective filter that use to remove negative outline noise is maximum filter, and it's use also in the computational of binary morphological dilation. maximum filter defined as:

$$
\operatorname{Maximum}(A)=\max [A(x+i, y+j)]
$$

Where the coordinate $x+I, y+j$ is defined over the image $A$ and the coordinate $I, j$ is defined over the mask $\mathrm{M}$. The mask $\mathrm{M}$ determines which pixels are used in the minimum calculation. 
In order to find the brightest points in an image:

First finds the maximum value in the area encompassed by the filter, and then reduces the pepper noise because of the maximum operation, finally the 100th percentile filter is max filter.

\section{Results}

The proposed methods have applied on different medical images. Some results are illustrated below. In each figure, we will see the original image, noisy image, noisy image filtered by minimum filter and noisy image filtered by maximum filter and noisy image filtered by median filter in both tow kinds of images.

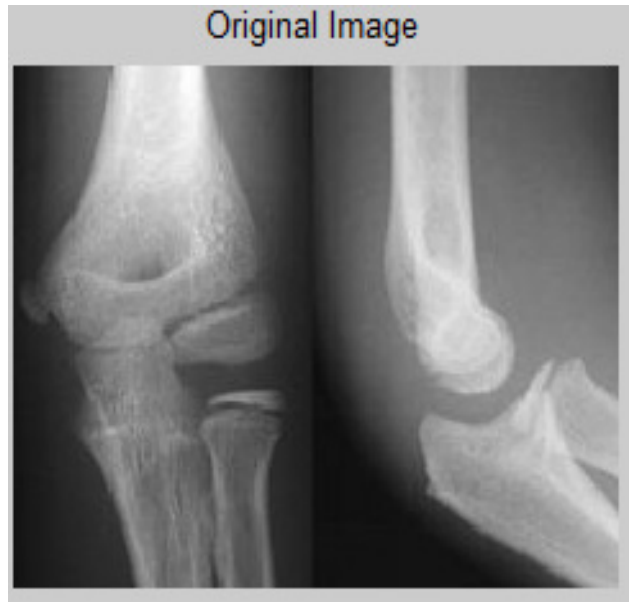

Figure 2. Original bone image

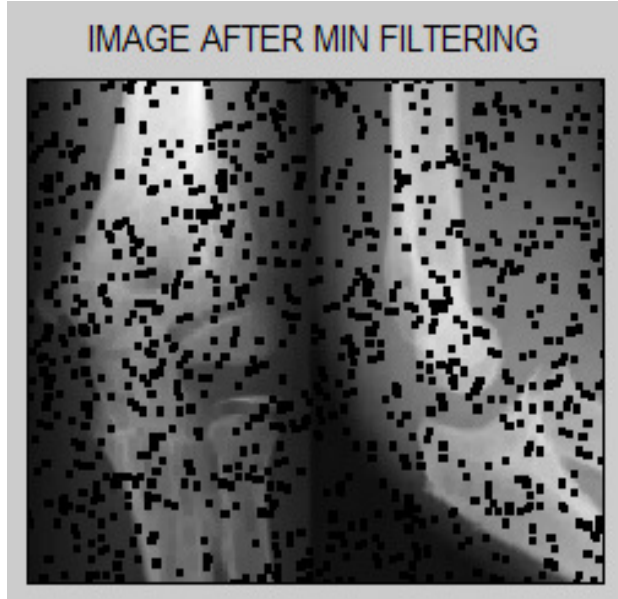

Figure 4.Bone image filtered by minimum filter

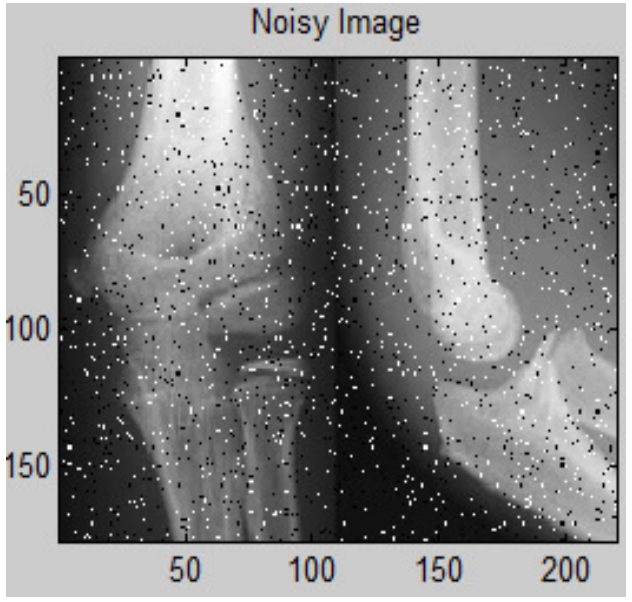

Figure 3. Noisy bone image



Figure 5.Bone image filtered by maximum filter 
International Journal of Computer Science \& Information Technology (IJCSIT) Vol 7, No 6, December 2015

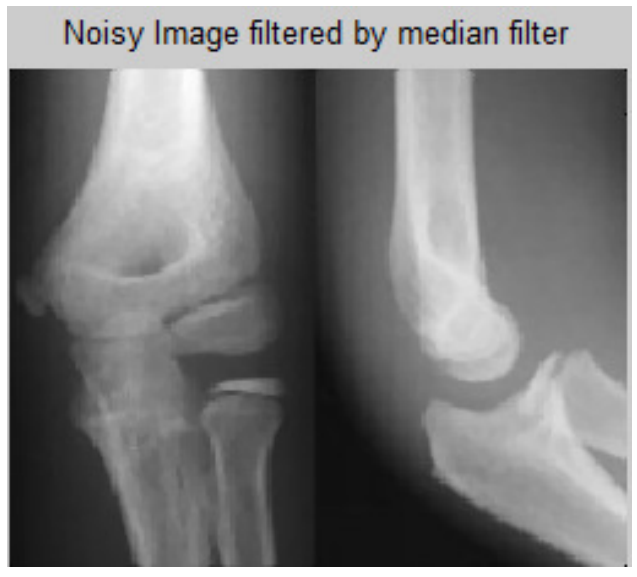

Figure 6.Bone image filtered by median filter



Figure 7.Original chest image

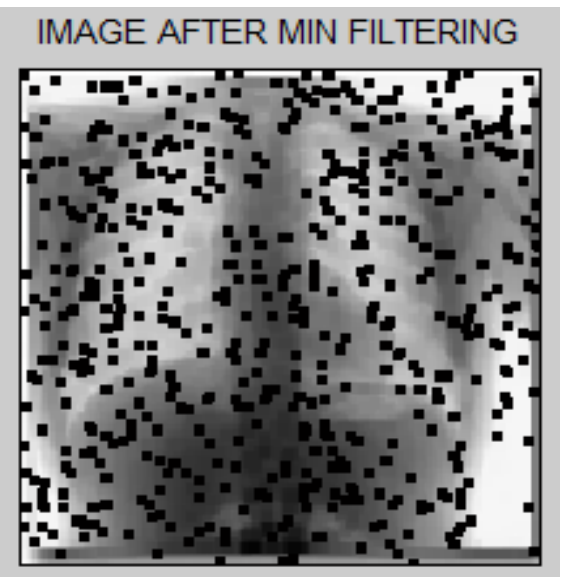

Figure 9. Noisychest image filtered by minimum filter

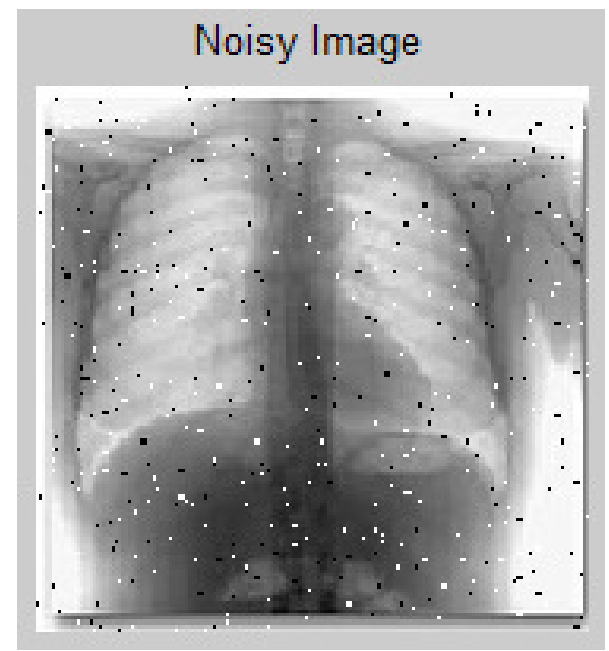

Figure 8.Noisychest image

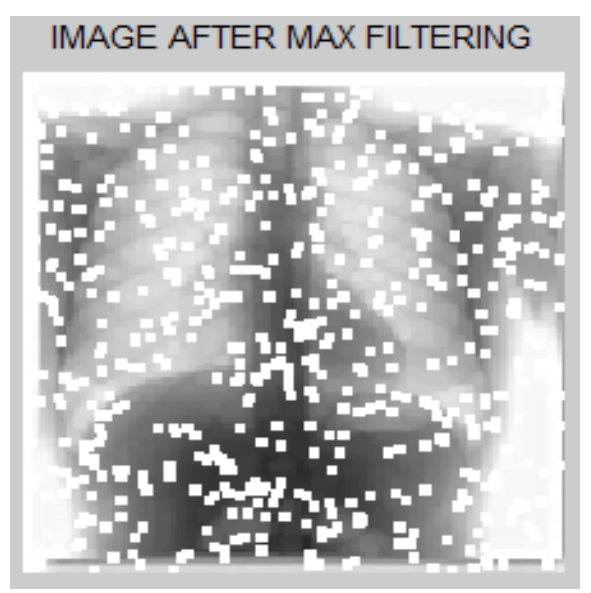

Figure 10.Noisychest image filtered by maximum filter 




Figure 11.Noisychest image filtered by median filter

\section{Conclusion}

In the field of medical image, generally various noises is added in image while acquiring from image input devices. Noises can change the properties or generate false output. Image filtering techniques remove noise from images so image processing algorithm can work effectively. Therefore image filtering is the main step in most image processing algorithm. In this paper the main aim is to remove the impulse noise from the medical image using non-linear filters. As we can see in the results aboveafter implementation, the median filter has the best results of removing noises of medical images.

\section{APPENDEX}






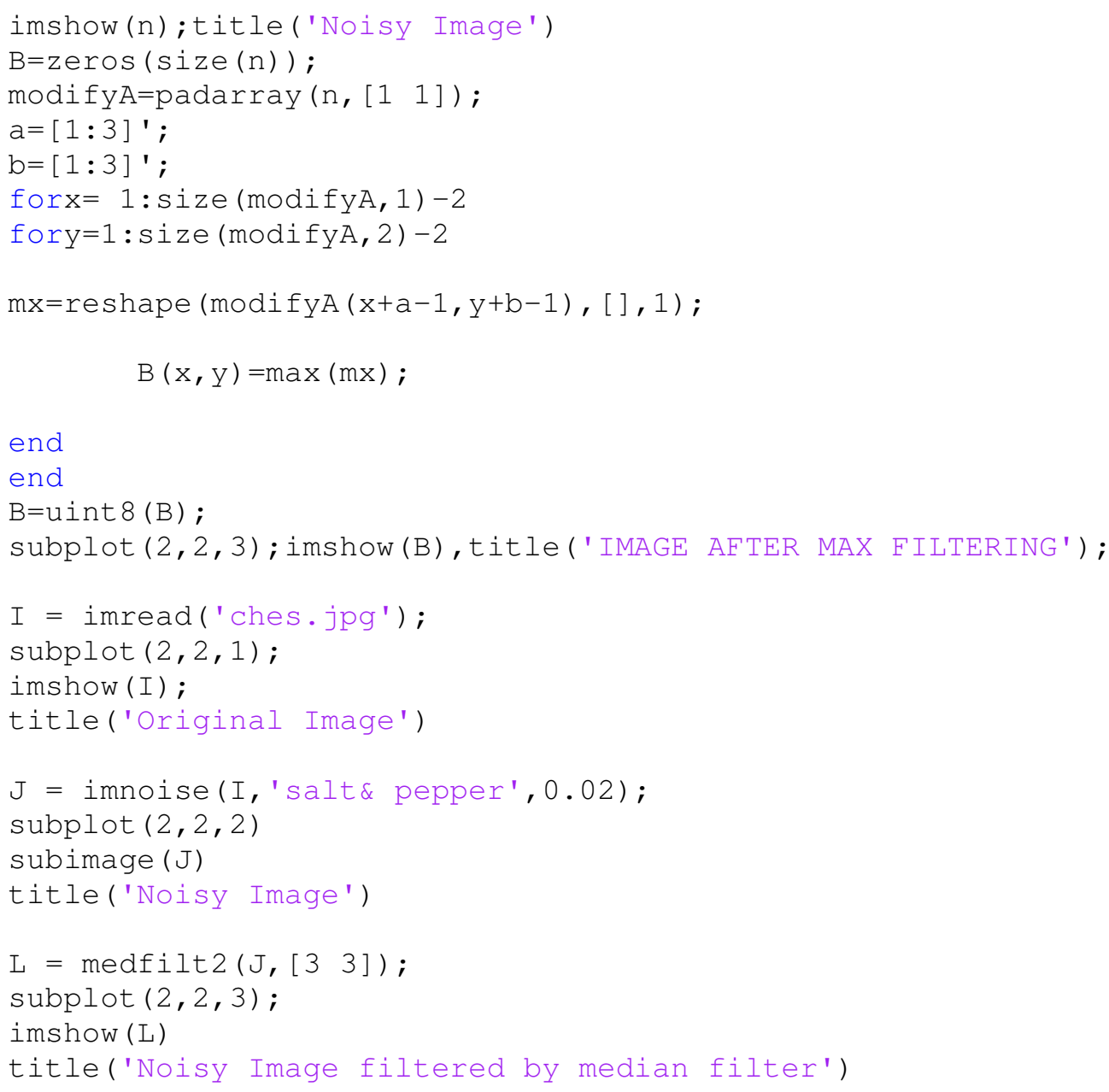

\section{REFERENCES}

[1] D.A. Forsyth and J. Ponce, Computer Vision - A Modern Approach, Prentice Hall, 2003

[2] Gregory d Abram, Parallel image generation, with anti aliasing and texturing. University of North Crolina Chapel Hill NC27599-3175

[3] Harley R Myler, Arthur R. Weeks, "The Pocket Handbook of Image Processing in C", Prentice Hall 1993

[4] Image Processing using Matlab. Second Edition. United States of America. Gatesmark Publishing.

[5] Mohamed Y. Adam, Mozamel M. Saeed and Al Samani A. Ahmed, "Medical Image Enhancement Application Using Histogram Equalization in Computational Libraries", http://www.ijcst.org/Volume6/Issue1/p2_6_1.pdf

[6] Mrozek, Bogumiła - Mrozek, Zbigniew 2001. Matlab 6; Poradnikużytkownika.

[7] Nayan Patel, Abhishek Shah, MayurMistry, "Astudy of Digital Image Filtering Techniques in Spatial Image Processing", International Conference on Convergence of Technology - 2014

[8] Ozimek, Agnieszka 2010b. Digital image processing. Materials from Lecture no. 7. Cracow. Cracow University of Technology

[9] Processing using Matlab. Second Edition. United States of America. Gatesmark Publishing

[10] Rafael C. Gonzalez and Richard E. Woods, Digital image processing, Third edition, Prentice Hall, 2008

[11] Rakesh M.R, Ajeya B, Mohan A.R,"Hybrid Median Filter for Impulse NoiseRemoval of an Image in Image Restoration",International Journal of Advanced Research in Electrical,Electronics and 
International Journal of Computer Science \& Information Technology (IJCSIT) Vol 7, No 6, December 2015

Instrumentation Engineering, Vol. 2, Issue 10, October 2013, http://ijareeie.com/upload/2013/october/8QHybrid.pdf

[12] Sigurdangenent, ericpichon, and allentannenbaum, "mathematical methods in medical image processing", http://www.math.wisc.edu/ angenent/preprints/medicalBAMS.pdf

[13] The MarthWorks 2009. Image Processing Toolbox 6 User's Guide. United States of America. The MathWorks.

[14] Warner and David, Digital Image processing - an analytic approach Prentice Hall ,2003 